\title{
EBIC-Guidelines for Management of Severe Head Injury in Adults
}

\author{
A. I. R. Maas', M. Dearden ${ }^{2}$, G. M. Teasdale ${ }^{3}$, R. Braakman ${ }^{4}$, F. Cohadon ${ }^{5}$, F. Iannotti ${ }^{6}$, A. Karimi ${ }^{7}$, \\ F. Lapierre ${ }^{8}$, G. Murray $^{9}$, J. Ohman ${ }^{10}$, L. Persson ${ }^{11}$, F. Servadei ${ }^{12}$, N. Stocchetti ${ }^{13}$, and A. Unterberg ${ }^{14}$ on $^{2}$ \\ behalf on the European Brain Injury Consortium
}

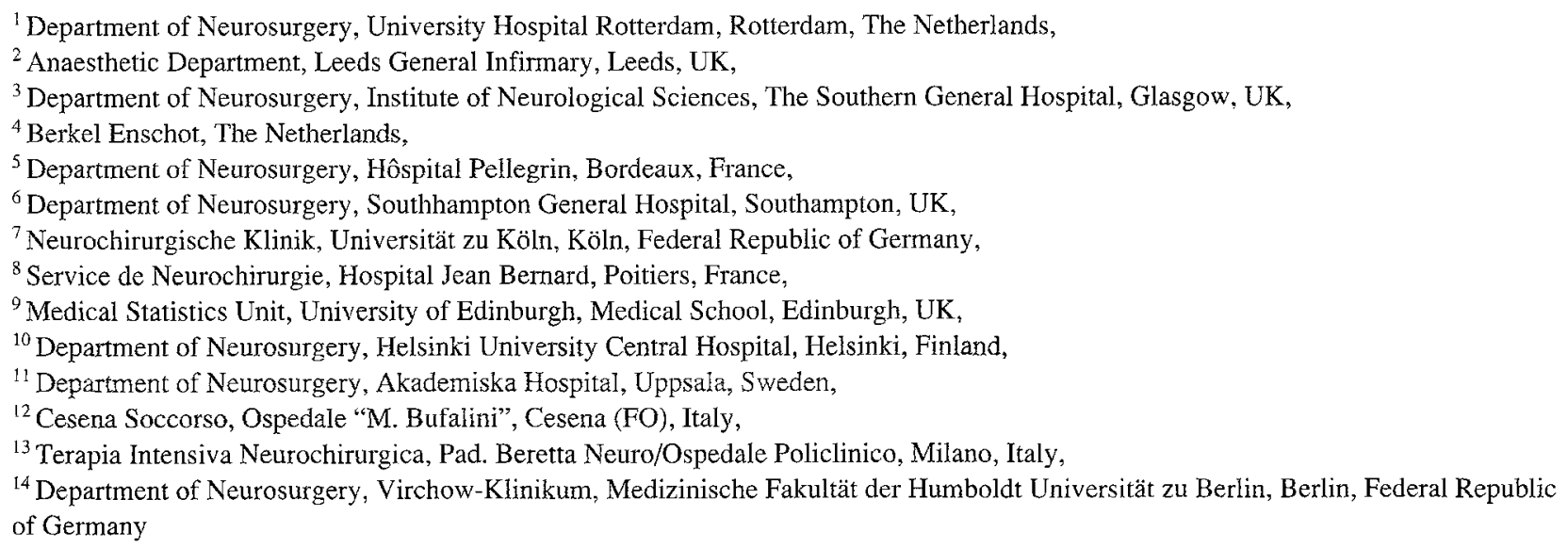

\section{Summary}

Guidelines for the management of severe head injury in adults as evolved by the European Brain Injury Consortium are presented and discussed. The importance of preventing and treating secondary insults is emphasized and the principles on which treatment is based are reviewed. Guidelines presented are of a pragmatic nature, based on consensus and expert opinion, covering the treatment from accident site to intensive care unit. Specific aspects pertaining to the conduct of clinical trials in head injury are highlighted. The adopted approach is further discussed in relation to other approaches to the development of guidelines, such as evidence based analysis.

Keywords: Brain injury; intensive care; secondary insults; clinical trials.

\section{Introduction}

\section{Head Injury: The Scope of the Problem}

Head injury is the most common cause of death in young adults in the western world, accounting for up to two thirds of in-hospital deaths and for a much larger proportion of lifelong disability after trauma [13]. Outcome and potential for successful rehabilitation after brain injury depend on the primary brain damage and on the quality of early management, adequate referral policy, prompt diagnosis and treatment of mass lesions as well as on preventing, limiting and treating processes leading to secondary damage. The importance of secondary delayed insults and of changes at the biochemical level inducing cerebral ischemia is increasingly recognized, and has led to the development of neuroprotective agents giving the potential for pharmacologic intervention. The design and conduct of clinical trials to investigate the efficacy of neuroprotective agents in the head injury population is proving to be a major challenge. Population imbalances in respect of basic prognostic variables, as well as inter-centre differences in approach to treatment complicate analysis [15]. Complete standardization of treatment is impractical, but a common "core approach" is desirable. Such approach should, as far as possible be based upon our understanding of general patho-physiologic mechanisms occurring in head injury, should reflect current treatment policies, and as such be open to wide acceptance. Some variation in 
treatment is inevitable, but methods that may potentially interact adversely with a new treatment under trial should be avoided.

The purpose of this paper is to describe the principles and practical details of guidelines for management of severe head injury evolved by the European Brain Injury Consortium (EBIC). This is an organisation of more than 100 European centres committed to research aimed at improving outcome in patients with head injury.

\section{The Importance of Secondary Insults and Basic Principles}

The mainstay of head injury management is based on the concept that little can be done about the primary brain injury, but that a lot can be done to minimize secondary brain injury because the duration and severity of secondary insults influence outcome. Secondary insults may be systemic or intracranial as listed in Tables 1 and 2.

Head injury management is focussed on preventing, detecting and correcting these secondary insults during initial care at the accident scene, in the casualty department, during transport between and within hospitals, during anesthesia and surgery and in the intensive care unit or neurosurgical ward. However,

Table 1. Systemic Secondary Insults

\begin{tabular}{|c|c|}
\hline Event & Main causes \\
\hline Hypoxemia & $\begin{array}{l}\text { hypoventilation } \\
\text { thoracic injury } \\
\text { aspiration, pneumonia } \\
\text { anaemia }\end{array}$ \\
\hline Hypotension & $\begin{array}{l}\text { hypovolemia } \\
\text { cardiac failure } \\
\text { sepsis } \\
\text { spinal cord injury }\end{array}$ \\
\hline Hypercapnia & respiratory depression \\
\hline Hypocapnia & $\begin{array}{l}\text { hyperventilation } \\
\text { spontaneous or induced }\end{array}$ \\
\hline Hyperthermia & $\begin{array}{l}\text { hypermetabolism } \\
\text { stress response } \\
\text { infection }\end{array}$ \\
\hline Hyperglycemia & $\begin{array}{l}\text { hypothermia, i.v. infusion of dextrose } \\
\text { stress response }\end{array}$ \\
\hline Hypoglycemia & inadequate nutrition \\
\hline Hyponatremia & $\begin{array}{l}\text { insufficient intake (hypotonic fluids) } \\
\text { excessive Sodium loss }\end{array}$ \\
\hline
\end{tabular}

Table 2. Intracranial Secondary Insults

\begin{tabular}{ll}
\hline Event & Cause \\
\hline $\begin{array}{l}\text { Raised intracranial } \\
\text { pressure and/or brain } \\
\text { shift }\end{array}$ & $\begin{array}{l}\text { mass lesion } \\
\text { vascular engorgement (brain swelling, } \\
\text { caused by vasodilation) } \\
\text { edema (increased brain water content) } \\
\text { hydrocephalus }\end{array}$ \\
Vasospasm & $\begin{array}{l}\text { traumatic subarachnoid hemorrhage? } \\
\text { cortical brain injury }\end{array}$ \\
Seizures & skull base fracture, \\
compound depressed skull fracture
\end{tabular}

the organisation of management in head injury varies greatly within and between European countries. Differences include facilities and standards of emergency care, the availability of investigational facilities in particular C T scan, subsequent intensive care regimens and patient discharge policies. The proportion of patients with a severe head injury admitted directly to a neurosurgical centre varies between $25 \%$ and $65 \%$. Some neurosurgical centres receive all patients with head injuries, others admit only those requiring neurosurgery. Nevertheless, the principles and basic standards of care in the management of head injured patients should be similar throughout Europe. In many fields of medicine adoption of clinical practice guidelines has been shown to improve treatment results [27]. Adopting guidelines for an improved referral policy in Scotland has substantially reduced mortality in patients with extradural hematoma and more complex intracerebral lesions [26].

The need for guidelines in the treatment of head injury, covering aspects of initial care, referral policies, neurosurgical consultations, operative indications and intensive care treatment has been recognized in many European countries and throughout the world. Much work has already been done on the primary assessment and resuscitation (ATLS ${ }^{\circledR}$-principles) [1], criteria for neurosurgical consultation, but less on intensive care management. In the United States a head injury guidelines task force was formed in 1993 by the joint section on Neurotrauma and Critical Care of the American Association of Neurological Surgeons and the Congress of Neurological Surgeons, supported by the Brain Trauma Foundation. The result is a detached document analyzing the evidence on which to base guidelines for the management of severe head injury [5]. Unfortunately, the 
main message has been to highlight the lack of rigorous evidence upon which clinical management is based. Thus, only four practice standards could be formulated, each explaining what should not be done, rather than what the actual treatment should be. It is therefore still necessary to develop an international consensus on practical guidelines for many aspects of head injury care. Such generally accepted strategies can then subsequently be adapted and tailored for national and local use, and form the basis for action in a specific context, as in a clinical trial.

\section{The EBIC Perspective to Guidelines in Head Injury}

The European Brain Injury Consortium (EBIC) was founded in 1994, to develop a strong clinical group to advise and to work in partner-ship with sponsors in order to ensure excellence in the design, conduct and analysis of clinical trials in head injury. It was felt essential to define general standards of management, so as to ensure similar approaches to conventional, medical and surgical management throughout the centres participating in clinical trials. It was agreed that these criteria should be pragmatic and would not for example insist on highly sophisticated levels of invasive monitoring, known not to be in general use. A working group drafted initial guidelines and following feedback from the Executive Committee of EBIC and subsequently from participating centres, the guidelines that follow were formulated.

\section{EBIC Guidelines for Management of Severe Head Injury in Adults: Pre-Hospital and Primary Hos- pital Care}

\section{Resuscitation, Stabilization}

Primary care is aimed at restoration and stabilization of adequate ventilation and circulation in accordance with ATLS ${ }^{\circledR}$ recommendations. Administration of high flow oxygen is advocated in all cases to prevent possible secondary hypoxic episodes.

A systolic blood pressure of $120 \mathrm{mmHg}$ should be achieved and maintained as soon as possible.

If the patient is unable to follow commands and if appropriate facilities and expertise are present, intubation and institution of IPPV should be performed before transport to the main Head Injury Trauma Centre. Ventilation is adjusted to achieve values of $\mathrm{PaCO}_{2}$, $4-4.5 \mathrm{kPa}(30-35 \mathrm{mmHg})$ and $\mathrm{PaO}_{2}>10 \mathrm{kPa}(75 \mathrm{mmHg})$ or $>95 \%$ saturation.

Documentation of:

- time of injury/nature/cause;

- neurological state: level of consciousness: GCS score (eye, motor and verbal separately), pupil size and reactivity, neurological deficit;
- extracranial injuries, trauma score.

Initial Survey of Injuries

$\mathrm{X}$-rays of chest, cervical spine, pelvis and other regions as indicated by clinical examination and mechanism of injury, but a comprehensive radiological examination should be delayed until the patient is fully stable and transferred to the regional neurotraumatological centre. Initial survey should include measures to exclude abdominal injuries. Emergency extracranial sugery may need to be performed before transport or $\mathrm{CT}$, if the patient remains haemodynamically unstable.

\section{Neurosurgical Centres}

Patients with a severe head injury, as well as those considered at increased risk for developing intracranial complications, should be treated in a centre that has CT available throughout 24 hours, neurosurgical service enabling prompt intracranial diagnosis and neurosurgical intervention, and dedicated ICU facilities. Although another specialist, experienced in the intensive care of neurotrauma, may be the physician primarily responsible, the neurosurgeon should be involved in care, even when there is not an operable lesion.

\section{Admission Care}

Reassessment, further resuscitation as above,

- Full survey of injuries, including measures to exclude abdominal injuries

- X-rays of chest, cervical spine, pelvis and other regions as indicated by clinical examination and mechanism of injury, but a comprehensive radiological examination should be delayed until the patient is fully stable.

- After stabilization, transport as soon as possible to CT with monitoring and supervision, anaesthetised on IPPV. It is emphasized that transport to $\mathrm{C} T$ should in the absence of rapid deterioration be performed only after optimal stabilization and that specialist care sould be provided during transportation.

- Emergency surgery for thoracic, abdominal or vascular lesions if of vital importance; preferably after brain CT. If the patient's condition does not permit CT before surgery, CT should be performed as soon as possible after surgery.

- Operable intracranial haematoma to be evacuated immediately after disclosure; consider the administration of mannitol and hyperventilation if there are signs of developing intracranial herniation.

\section{ICU Care}

Monitoring and General Care

Minimal monitoring requirements include $\mathrm{ECG}, \mathrm{SaO}_{2}$, invasive arterial blood pressure, temperature and End Tidal $\mathrm{CO}_{2}$ if ventilated.

Maintain: $\mathrm{SaO}_{2}>95 \%$, Mean arterial blood pressure $>90 \mathrm{mmHg}$.

Give IV fluids to maintain normovolaemia and normal blood chemistry; there is no indication for fluid restriction, as treatment of cerebral edema. In selected cases with severe concomitant pulmonary injury/edema some fluid restriction may be appropriate. 
Central venous pressure monitoring is recommended to ensure normovolemia.

If ICP is monitored then there should also be continuous monitoring of $\mathrm{ABP}$ and calculation of $\mathrm{CPP}$.

Ventilatory parameters: while on IPPV, adjust ventilation to maintain:

$\mathrm{PaO}_{2}>13 \mathrm{kPa}(100 \mathrm{mmHg})$

$\mathrm{PaCO}_{2} 4-4.5 \mathrm{kPa}(30-35 \mathrm{mmHg})$

Early institution of nutrition by enteral feeding. General care is aimed at maintaining normal 'milieu interne' avoiding hyperthermia, hyperglycaemia, preventing and promptly treating secondary complications.

Consider follow-up CT next day or earlier if clinically indicated. A single early initial CT often does not disclose the full extent of intracranial injuries.

\section{(Further) Extracranial Surgery}

There is no general consensus on the timing of more elective orthopaedic or craniofacial surgery in the absence of a life threatening disorder. Some clinicians advocate early internal fixation of limb fractures or faciomaxillary deformaties; others advocate delayed surgery. If early surgical repair is chosen there should be full cardiovascular monitoring, and preferably also ICP monitoring; procedures should be carried out in accordance with neuroanaesthesia principles, e.g., cerebral vasodilation and episodes of hypotension must be avoided. Preoperative evaluation of the coagulation system is recommended. During surgery monitoring of the neurological condition (pupils) and preferably also ICP and/or neurophysiologic monitoring is advocated. However, such monitoring procedures may fail to identify the development of focal mass lesions, with midline shift and for this reason early prolonged extracranial surgery for non lifethreatening injuries should be avoided.

\section{Management of CPP and ICP}

ICP therapy is only definitely indicated if raised ICP has been demonstrated by monitoring, if there is CT evidence of increased ICP (e.g., absent/compressed basal cisterns) or clinical signs of developing intracranial herniation. Treatment should not only be aimed at reducing ICP, but especially at restoring CPP to appropriate levels $(60-70 \mathrm{mmHg})$. The precise level of ICP above which treatment should be instituted has not been established, but generally ICP elevations above $20-25 \mathrm{mmHg}$ should be treated unless other clear local policies have been identified. In the first few days after trauma more active treatment is indicated than in the later post-traumatic phase. There is no consensus whether patients should be nursed flat or with the head up to a maximum of $30^{\circ}$ elevation.

Before initiating treatment directed at ICP, check for monitor malfunction, remediable extracranial disorders $\left(\mathrm{PaCO}_{2}, \mathrm{BP}\right.$ etc. $)$.

Accepted methods of management of ICP and CPP are:

- Sedation, analgesia and mild to moderate hyperventilation $\left(\mathrm{PaCO}_{2} 4-4.5 \mathrm{kPa}, 30-35 \mathrm{mmHg}\right.$ )

- Volume expansion and inotropes or vasopressors when arterial blood pressure is insufficient to maintain CPP in a normovolaemic patient
- Osmotic therapy: preferably mannitol given repeatedly in bolus infusions, or as indicated by monitoring. Serum osmolarity should be maintained $\leq 315$. Other agents, such as Glycerol or Sorbitol are not advocated. If osmotherapy has insufficient effect, Furosemide (Lasix) can be given additionally.

- CSF drainage

- If these methods fail more intensive hyperventilation $\left(\mathrm{PaCO}_{2}<30 \mathrm{mmHg}\right)$, preferably with monitoring of cerebral oxygenation to detect cerebral ischemia, for instance by jugular oxymetry. Altematively the use of barbiturates, inducing increased sedation may be considered.

Other ICP therapy is considered to be experimental and should not be instituted in patients enrolled into studies of other forms of treatment. There is no established indication for steroids in the management of acute head injury.

\section{Operative Therapy (Timing, Indications)}

- A surgically significant epidural haematoma, or acute subdural haematoma should be evacuated immediately upon detection.

- For small haemorrhagic contusions or other small intracerebral lesion: a conservative approach is generally adopted; but operation should be considered urgent for large intracerebral lesions with high or mixed density on CT scan.

Specific indications for operation include:

a) clinical deterioration;

b) size: $>1 \mathrm{~cm}$ thick extracerebral clot, $>25-30 \mathrm{ml}$ intracerebral haematoma;

c) midline shift $>5 \mathrm{~mm}$;

d) emlargenent of contralateral ventricle (temporal horn);

e) obliteration of basal cisterns/third ventricle;

f) raised or increasing ICP.

- Depressed skull fracture: operation is definitely indicated only if it is a compound (open) fracture (not over sagittal sinus) or if the fracture is so extensive that it causes mass effect.

- Closed depressed skull fractures are usually treated conservatively, but operation may be appropriate in selected cases to reduce mass effect or correct defigurement.

- Decompressive craniotomy: may be considered in exceptional situations.

The foregoing guidelines emerge from consideration of basic biological and pathophysiological principles, informed and refined by published evidence and practical experience.

\section{Discussion}

\section{Early Management of Head Injury}

An accurate documentation of nature, cause and time of injury, trauma score, Glasgow Coma Scale and pupil reactivity are important, both for clinical reasons and from the perspective of a potential trial. The nature and cause of injury can alert physicians to the presence of different intra- and extracranial injuries. Often too little attention is paid to the observations of emergency medical personnel. The initial 
survey of injuries should be limited and focus on those that may have immediate consequences for treatment, such as intra-thoracic, abdominal, pelvic or spinal injuries.

Accurate documentation of the exact time of injury is important, because it is believed that a time window exists between the time of injury and administration of a clinical trial intervention, beyond which efficacy may be reduced. In clinical trials the "post resuscitation GCS sum score" is often used to select a population of head injured patients. However, in many countries where emergency physicians or technicians with extended skills are involved in the EMS-system many patients are intubated and ventilated on the scene of the accident and arrive in the hospital already paralysed and ventilated. In these situations an accurate GCS from the scene of the accident is the next best alternative and essential when considering possible enrollment of patients in clinical trials. If after stabilization and investigation there remains some doubt on the severity of injury, e.g. if the CT scan does not show features that confirm intracranial damage, it may be appropriate to allow sedation and paralysis to reverse, in order to re-assess the severity of injury.

Cerebral ischemia is considered the central mechanism leading to secondary brain damage in patients after brain trauma. In patients dying from head injury ischemic changes can be demonstrated in more than $90 \%$ of cases $[10,19]$.

Clinical studies have shown critically reduced cerebral blood flow [4] following head injury and the presence of ischemia at the tissue level has been further demonstrated in studies where local brain tissue $\mathrm{pO}_{2}$ [20] or jugular oxygen saturation [21] was continuously monitored or microdialysis [17] performed. Although debate exists over the causes of low CBF after head injury, the consequences of secondary ischemic damage are evident, and the adverse influence of further systemic hypoxic and hypotensive insults has been well documented $[6,8,14]$. Preventing and treating ischemia after injury is a major goal in trauma care and immediate restoration and stabilization of adequate ventilation and circulation, in agreement with ATLS $^{\circledR}$ standards are paramount.

The question whether emergency medical service should be limited to "scoop and run" or whether a policy of "stay and play", i.e., optimal resuscitation at the scene of the accident should be preferred, has not been addressed by EBIC because this depends on local organisational aspects of the EMS system as well as on the distance and transport time to local district general hospital or neurotraumatological referral unit. Studies have shown a high incidence of ischemic insults at the scene of the accident and would thus seem to favour optimal resuscitation at the scene of the accident [24].

Although moderate hyperventilation may be beneficial in the presence of raised intracranial pressure, excessive hyperventilation should be avoided, because of the risk of enhancing vasoconstriction, further compromising $\mathrm{CBF}$. In the presence of prehospital shock, defined as a systolic blood pressure $\leq 90 \mathrm{mmHg}$ mortality is increased two or threefold [6]. The level of $90 \mathrm{mmHg}$ as reported in various studies would appear the absolute minimum below which brain circulation is compromised. However, the risk of developing cerebral ischemia exists at higher levels of blood pressure, especially when raised intracranial pressure may be present. For this reason a systolic blood pressure of at least $120 \mathrm{mmHg}$ is recommended for the prehospital situation. Measuring diastolic or mean arterial blood pressure is not practical in the prehospital situation. For in-hospital situations the target should be a mean arterial blood pressure of at least $90 \mathrm{mmHg}$, depending also on age of patient and information concerning pretrauma normal blood pressure.

\section{Referral Policy and Admission Care in Neurotrauma- tological Centre}

Patients with severe head injury treated in major neurotraumatological centres with considerable expertise and experience in the treatment of such patients have better outcome results than in centres with fewer admissions [23]. Concentrating the care of neurotrauma will improve outcome and facilitate research on new methods of monitoring and treat-

Table 3. Risk of an Operable Intracranial Haematoma in Head Injured Patients

\begin{tabular}{llll}
\hline GCS & Risk & Other features & \multicolumn{2}{c}{ Risk } \\
\hline 15 & \multirow{2}{*}{1 in 3,615 } & None & 1 in $: 31,300$ \\
& & PTA & $: 6,700$ \\
& & Skull fracture & $: 81$ \\
& & Skull fracture and PTA & $: 29$ \\
$9-14$ & \multirow{2}{*}{1 in 51} & No fracture & 1 in $: 180$ \\
& & Skull fracture & $: 5$ \\
$3-8$ & 1 in 7 & No fracture & 1 in $: 27$ \\
& & Skull fracture & $: 4$ \\
\hline
\end{tabular}

Data derived from Teasdale et al. 1990 [25]. 
ment. A multidisciplinary approach is essential, but needs to be co-ordinated by a specialist experienced in evaluation and intensive care of neurotrauma. Facilities for 24 hours CT and neurosurgical services are a prerequisite for prompt intracranial diagnosis and neurosurgical intervention if appropriate. The presence or absence of a skull fracture and the level of consciousness are important when considering the risk of harbouring a potentially operable intracranial hematoma (Table 3 ). Based on this evidence it may be concluded that CT examination of the head is required in patients with one or more of following characteristics:

- with severe and moderate head injury;

- who are fully conscious with skull fracture;

- in whom confusion persists after initial resuscitation;

- in an unstable systemic state precluding transfer to neurosurgical unit;

- with an uncertain diagnosis.

The EBIC-guidelines deliberately avoid specifying which patients should be referred to neurosurgery as possibilities for receiving such patients depend on local organisational aspects. This topic has been addressed in Britain and indications for referral specified by the Society of British neurological surgeons (Table 4).

\section{Transportation}

Patients who are admitted to a district general hospital and require transport to a neurotraumatological

Table 4. Indications for Transfer to Neurosurgical Unit. Society of British Neurological Surgeons 1996

I Urgent referral, preliminary computed tomography not necessary

Coma persisting after resuscitation

Deteriorating consciousness or progressive neurological deficit

Open injury: compound fracture of vault or base of skull Patient fulfills criteria for CT in general hospital but this cannot be performed urgently

II Referral after computed tomography in a general hospital

Abnormal scan

Normal scan but patient's progress is unsatisfactory

(Consult Neurosurgeon on images transferred electronically) centre are at considerable risk of suffering secondary insults during transfer. Numerous studies highlight the adverse effects of failing to adequately resuscitate and monitor head injury patients during inter- and intrahospital transport [2, 9]. Minimal monitoring requirements for transport include ECG, pulse oximetry to measure oxygen saturation and blood pressure. All patients in coma, those with multiple injuries and those who are restless should be anesthetized and artificially ventilated for transport. Short acting agents, e.g., Midazolam, Alfentanyl, Atricurium, that are readily reversable to allow assessment are to be preferred.

Invasive monitoring of blood pressure and preferably also End Tidal $\mathrm{CO}_{2}$ is advocated. The patient should be transferred with a trained escort, preferably an anesthetist, with suitable drugs including anesthetic drugs, anticonvulsants, mannitol and emergency resuscitation drugs. No patient should be transferred until fully monitored an resuscitated.

On admission to the neurotraumatological centre the emphasis should again be on adequate ventilation and circulation. The desire to rush patients for CTexamination before optimal stabilization has been achieved must be resisted. Only in the presence of rapid neurological deterioration or signs of developing herniation is urgent CT-examinaiotn required but only after resuscitation. Optimal monitoring and supervision should be available during intra-hospital transport and CT-examination.

\section{ICU-Care}

Artificial ventilation is employed in most centres for patients with a GCS $\leq 8$, patients with multiple injuries and patients with evidence of brain swelling on CT-scan. Sedation and analgesia without paralysis are increasingly used, after the first 12 hours of intensive care. The routine use of paralysis increases complications [12].

Ventilation is adjusted to $\mathrm{PaO}_{2} \geq 13 \mathrm{kPa}$ and $\mathrm{PaCO}_{2}$ to $4.5 \mathrm{kPa}$. Volume resuscitation is continued, aiming at a mean arterial blood pressure $\geq 90 \mathrm{mmHg}$, since low blood pressure, rather than high intracranial pressure, is the most frequent cause of inadequate cerebral perfusion pressure [14]. Mean arterial blood pressure should be kept above $90 \mathrm{mmHg}$ with intravenous fluids and if necessary drugs that raise blood pressure.

General care includes early institution of enteral feeding, maintenance of a normal "milieu interne", 
avoiding high temperature and high blood sugar levels. Both hyperthermia and hyperglycemia have been identified as cause of secondary insult [14]. Intravenous fluids are given to maintain normal biochemistry and normal blood volume. Epileptic fits are controlled with Diazepam, Phenytoin or Sodium Valproate. Profylactic antriconvulsant therapy is rarely used in Europe but in American neurosurgical centres Phenytoin is routinely administered, even though no evidence exists for its efficacy in preventing late posttraumatic epilepsy [5].

In most neurotraumatology centres intensive care management of head injured patients involves monitoring of invasive blood pressure and ICP continuously with an aim of keeping CPP above 60 to $70 \mathrm{mmHg}$ and ICP $\leq 20$ to $25 \mathrm{mmHg}$. Higher levels of ICP may be tolerated after the first 72 hours of brain injury. The American Brain Injury Consortium strongly advocate the use of ICP monitoring in all clinical trial centres because of the frequent occurrence of raised ICP after brain trauma (about $70 \%$ of head injured patients managed on the ICU) and to facilitate early detection of mass lesions, improved rationale for ICP therapy and assistance in determining prognosis. EBIC considers ICP measurement desirable, and a survey of participating centres indicated that in 1996 it was used in over $70 \%$ of patients, but acknowledges that not all European centres routinely practise ICP monitoring. Some centres only monitor ICP in high risk groups, for example comatose patients with an abnormal CT scan or patients over 40 years with abnormal motor posturing of systolic blood pressure $<90 \mathrm{mmHg}$ despite normal CT scan [16]. However, recent studies suggesting an improved outcome for patients managed with CPP above $70 \mathrm{mmHg}$ [18] may lead to even greater use of ICP monitoring in the future.

ICP therapy is only indicated when raised ICP has been measured, is suspected on basis of CT examination (i.e., absence of third ventricle or compression of basal cisterns) or there are clinical signs of brainstem herniation. Monitor malfunction and extracranial causes, such as low blood pressure, low arterial $\mathrm{pO}_{2}$, high $\mathrm{PaCO}_{2}$ and fighting the ventilator should be eliminated prior to commencing ICP treatment. The accepted treatment modalities for management of CPP and ICP reflect the so-called staircase approach. Institution of targetted therapy remains experimental since additional monitoring and analysis is required to deternine the cause of raised intracranial pressure, The results of multimodality monitoring protocols performed in many high level traumacentres will hopefully aid in this respect in the future. If the patient has an indwelling intraventricular ICP monitor drainage of CSF may be used to reduce ICP. In most patients however the treatment of choice is sedation, analgesia, moderate hyperventilation and intravenous infusion of 0.25 to 0.5 gram per kilogram mannitol $20 \%$ over 15 to 20 minutes repeated as necessary. Blood osmotic pressure must be monitored and serum osmolarity kept below $315 \mathrm{~m}$ osm. Furosemide, $0.4 \mathrm{mg} / \mathrm{kg}$ may be administered with Mannitol and blood volume maintained with intravenous fluids, usually colloids. Aggressive hyperventilation to lower $\mathrm{PaCO}_{2}$ levels below $4 \mathrm{kPa}$ may be employed provided monitoring of cerebral oxygenation is instituted, usually with a catheter in the jugular vein, just below the skull to measure brain oxygen extraction.

Vasoconstrictors or inotropes to restore blood pressure in a patient with normal blood volume may be used, with appropriate CVP-monitoring. To increase blood pressure to supranormal levels is considered experimental therapy, both by the American and European Brain Injury Consortium. Barbiturates should be considered if ICP is refractory to other treatment modalities. Other forms of ICP therapy, for which evidence of benefit in outcome is lacking, such as hypothermia and infusion of Indomethacin or Ergotamine are considered experimental at present and should not be used in patients enrolled into clinical trials of cerebral neuroprotective agents after brain injury, because of the potential interaction with clinical parameters such as blood pressure.

When conducting clinical trials on neuroprotective drugs aspects of safety are as important as efficancy. In initial phase II studies, the major problems of the new treatment should have been identified, but as more data are collected in the phase III efficacy studies the potential occurrence of untoward and often unsuspected events should be documented. Non proven therapies, especially those that may enhance the risk to the patient or have interactions with a treatment under investigation should be avoided and management should be based on standard accepted approaches.

Treatment for raised ICP should only be continued if it is effective in decreasing ICP and/or increasing CPP. Follow-up CT-examination is important because lesions may increase or new lesions develop, mainly within 12 to 24 hours of trauma [22, 28]. For this reason routine follow-up CT-examination within 24 
hours of trauma is recommended, but should be performed earlier if the clinical situation so dictates.

There is no convincing evidence that steroids are effective after brain injury [5] and from a clinical trial perspective their routine administration is discouraged. As yet there is no convincing evidence that the administration of calcium antagonists benefit the overall population of patients with head injury. A retrospective analysis of the subgroup of patients with traumatic subarachnoid hemorrhage showed a benefit in those given Nimodipine [7] in one trial, but in another this was absent [3]. A subsequent small prospective study [11], which included only patients with traumatic subarachnoid hemorrhage, but whose clinical severity varied, showed benefit. This needs replication in more extensive prospective series. As yet, the evidence for efficacy of Nimodipine, albeit pomising, is not considered compelling enough to warrant the routine administration of this drug in patients with traumatic subarachnoid hemmorhage.

\section{The Future of the Guideline Movement}

Consensus already exists among major neurotraumatologic centres throughout the world on basic management of patients with head injury; this is reflected by the similarity of general conclusions and recommendations found in the US guidelines [5] and these EBIC guidelines, despite different approaches adopted (evidence based in the former and consensus and expert opinion in the latter). However, not all patients with severe head injury are treated in a major neurotraumatologic centre. It is to be hoped that acceptance and implementation of guidelines by centres not already involved in head injury research will promote the quality of care and improve outcome, as awareness is increased about which patients are at risk for developing operable lesions, and the importance of preventing and treating secondary insults is recognized. General guidelines should not differ between countries or across continents. The publication containing the North American guidelines represents an excellent overview of the evidence underpinning approaches to head injury management, and as such can be viewed as an invaluable reference manual. The EBIC guidelines presented are of a more pragmatic nature, originating from the desire to promote coherent treatment in centres participating in clinical trials, recognizing that data to provide an evidence-based approach to all aspects of management is, and may always be, unavailable. The two docu- ments can be viewed as complimentary. In individual countries aspects of local organisation, e.g., concerning primary care and emergency transport systems mean that the general guidelines may need to be further elaborated on and tailored towards the local situation. In this regard collaboration with other specialists involved in the field of traumatology, especially emergency physicians, traumatologists and critical care specialists is needed. General acceptance of such guidelines and implementation of local protocols will represent a major accomplishment, that will hopefully improve outcome following brain injury by optimising resources, organisation and current methods and enabling research into the extra benefit gained from new approaches.

\section{References}

1. American College of Surgeons (1993) Advanced Trauma Life Support Course for Physicians, Student Manual. ACS, Chicago

2. Andrews PJD, Piper IR, Dearden NM, Miller JD (1990) Secondary insults during intrahospital transfer of head injured patients. Lancet $327-330$

3. Bailey I, Bell A, Gray J et al (1991) A trial of the effect of nimodipine on outcome after head injury. Acta Neurochir (Wien) 110: 97-105

4. Bouma GJ, Muizelaar JP, Stringer WA et al (1992) Ultra early evaluation of regional cerebral blood flow in severely head injured patients using xenon enhanced computerized tomography. J Neurosurg 77: 360--368

5. Bullock R, Chesnut RM, Clifton C et al (1996) Guidelines for the management of severe head injury. $J$ Neurotrauma 13 : 643-734

6. Chesnut RM, Marshall LF, Klauber MR et al (1993) The role of secondary brain injury in determining outcome from severe head injury. J Trauma 34: 216-222

7. The European Study Group on Nimodipine in severe head injury (1994) A multicentre trial of the efficacy of nimodipine on outcome after severe head injury. J Neurosurg 80: 797-804

8. Fearnside MR, Cook RJ, McDougall P, McNeal RJ (1993) The Westmead head injury project outcome in severe head injury. A comparative analysis of prehospital, clinical and CT variables. Br J Neurosurg 7: 267-279

9. Gentleman D (1992) Causes and effects of systemic complications among severely head injured patients transferred to a neurosurgical unit. Int Surg 77: 297-302

10. Graham DI, Ford I, Adams JH et al (1989) Ischemic brain damage is still common in fatal non missile head injury. $\mathrm{J}$ Neurol Neurosurg Psychiatry 52: 346-350

11. Harders A, Kakarieka A, Braakman R et al (1996) Traumatic subarachnoid hemmorhage and its treatment with Nimodipine. J Neurosurg 85: 82-89

12. Hsiang JK, Chesnut RM, Crisp CB et al (1994) Early routine paralysis for intracranial pressure control in severe head injury: is it necessary. Crit Care Med 22: 1471-1476

13. Jennett B (1991) Epidemiology of severe head injury: socioeconomic consequences of avoidable mortality and morbidity. 
In: Scriabine A, Teasdale GM, Tettenborn D, Young W (eds) Nimodipine. Pharmacological and clinical results in cerebral ischemia. Springer, Berlin Heidelberg New York Tokyo, pp 225-233

14. Jones PA, Andrews PA, Midgley S et al (1994) Measuring the burden of secondary insults in head injured patients during intensive care. J Neurosurg Anesthesiol 6: 4-14

15. Marshall LF, Marshall SB (1995) Pitfalls and advances from the international Tirilazad trial in moderate and severe head injury. J Neurotrauma 12: 929-932

16. Narayan RK, Kishore PR, Becker DP et al (1982) Intracranial pressure: to monitor or not to monitor? A review of our experience with severe head injury. J Neurosurg 56: 650-659

17. Persson L, Hillered L (1992) Chemical monitoring of neurosurgical intensive care patients using intracerebral microdialysis. J Neurosurg 76: 72-80

18. Rosner MJ, Rosner SD, Johnson AH (1995) Cerebral perfusion pressure: management protocol and clinical results. $J$ Neurosurg 83: 949-962

19. Ross DT, Graham DI, Adams JH (1993) Selective loss of neurons from the thalamic reticular nucleus following severe human head injury. J Neurotrauma 10: 151-165

20. Santbrink H van, Maas AIR, Avezaat CJJ (1996) Continuous monitoring of partial pressure of brain tissue oxygen in patients with severe head injury. Neurosurgery $38: 21-31$

21. Sheinberg M, Kanter JM, Robertson CS et al (1992) Continu- ous nomitoring of jugular venous oxygen saturation in head injured patients. J Neurosurg 76: 212-217

22. Servadei F, Nanni A, Nasi MT et al (1995) Evolving brain lesions in the first 12 hours after head injury: analysis of 37 comatose patients. Neurosurgery $37: 1-9$

23. Smith R, Frateschi L, Slown E et al (1990) The impact of volume on outcome in seriously injured trauma patients: two years experience of the Chicago trauma system. J Trauma 30: 1066-1076

24. Stocchetti N, Furlan A, Volta F (1996) Hypoxemia and arterial hypotension at the accident scene in head injury. J Trauma 40(5): 764-767

25. Teasdale G, Murray G, Anderson E et al (1990) Risks of an acute intracranial hematoma in children and adults: implications for managing head injury. B M J 300: 363-367

26. Teasdale G (1991) The treatment of head trauma: implications for the future. J Neurotrauma 8 [Suppl. 1]: 53-58

27. Wolf SH (1993) Practice guidelines: A new reality in medicine. Arch Intern Med 153: 2646-2655

28. Yamaki T, Hirakawa K, Uekuchi T et al (1990) Chronological evaluation of acute traumatic intracerebral hematoma. Acta Neurochir (Wien) 103: 112-115

Correspondence: A. I. R. Maas, Department of Neurosurgery, University Hospital Rotterdam, Dr. Molewaterplein 40, 3015 GD Rotterdam, The Netherlands. 